ÉGYPTE

monde arabe

\section{Égypte/Monde arabe}

4-5 | 2001

L'Égypte dans le siècle, 1901-2000

\title{
Le chômeur et le prétendant
}

Les maux de la jeunesse ou l'impossible passage à l'âge adulte

\section{Karine Tourné}

\section{(2) OpenEdition}

\section{Journals}

Édition électronique

URL : https://journals.openedition.org/ema/876

DOI : $10.4000 /$ ema.876

ISSN : 2090-7273

Éditeur

CEDEJ - Centre d'études et de documentation économiques juridiques et sociales

Édition imprimée

Date de publication : 30 juin 2001

Pagination : 191-206

ISBN : 2-87027-963-9

ISSN : 1110-5097

Référence électronique

Karine Tourné, «Le chômeur et le prétendant », Égypte/Monde arabe [En ligne], 4-5 | 2001, mis en ligne le 08 juillet 2008, consulté le 07 juillet 2022. URL : http://journals.openedition.org/ema/876 ; DOI : https://doi.org/10.4000/ema.876

Ce document a été généré automatiquement le 7 juillet 2022.

Tous droits réservés 


\title{
Le chômeur et le prétendant
}

\author{
Les maux de la jeunesse ou l'impossible passage à l'âge adulte
}

\author{
Karine Tourné
}

1 D'une génération à l'autre, l'entrée dans la vie active ou le passage à l'âge adulte ne répondent pas aux mêmes modalités ni aux mêmes moments. Ce constat souligne communément que la jeunesse est un «fait social instable » (de Singly, Gauthier, 2000), dont les âges tout autant que les images se construisent selon le contexte historique, économique et social. Entre hier et aujourd'hui, la jeunesse n'est donc plus « ce qu'elle était », tant au niveau des âges que des expériences et situations qui la distinguent des autres âges de la vie. En mobilisant deux corpus différents, celui d'un terrain et celui de l'analyse de la presse, et en privilégiant l'aller-retour entre récits de jeunesse et représentations de la jeunesse', nous nous attachons aux processus par lesquels, d'une époque à l'autre, sont produits de nouveaux âges et figures de la jeunesse.

2 L'enquête menée dans trois quartiers populaires du Caire ${ }^{2}$ auprès de deux générations, celle des parents, nés entre 1940 et 1950, et celle de leurs enfants, nés entre 1970 et 1980, met en évidence d'importantes différences dans l'expérience de la jeunesse. Les récits collectés ${ }^{3}$ (Dubar et Demazière, 1997) traduisent, au-delà des nouvelles modalités du projet matrimonial et professionnel, la diversité des formes d'expériences (Dubet, 1994) et de rythme des biographies (Beaud, 1996, Lautier, 1995). À la cohérence des enchaînements se sont substituées la révocabilité des situations et la désynchronisation des passages. La quête de l'autonomie prend alors le sens d'une navigation, dont les aléas donnent à voir de nouveaux rapports à l'emploi et de nouveaux modes de vie face à l'injonction au mariage. Ils amènent aussi questionner les modalités du passage à l'âge adulte (et plus largement des rapports entre les âges sociaux).

3 La mise en scène, notamment par les médias, de ces ajustements professionnels et matrimoniaux alimente au contraire les discours sur la déviance, l'anomie ou l'extrémisme. Ainsi, l'état des lieux sur la jeunesse d'aujourd'hui renvoie au vocabulaire de la crise, à l'actualité des "maux de la jeunesse égyptienne » (Meijer, 2000) : le chômage, l'obstacle du logement et la difficile réalisation du mariage diffèrent l'intégration professionnelle et sociale des jeunes et la transforment en une « insertion problématique » (Marie, 1997). Tant pour le sens commun et les médias que les experts, 
cette crise d'insertion résumerait l'expérience de la jeunesse dans les années 1990. Le temps de la jeunesse, comme celui de la quête de l'autonomie, s'allongerait alors jusqu'à produire de nouveaux âges de la jeunesse. Décrivant la jeunesse comme impasse, les médias et les politiques publiques contribuent ainsi à l'émergence de nouvelles figures jeunes': celle du chômeur diplômé et du prétendant.

La quête de l'autonomie : de la trajectoire à la navigation

Dans une perspective en termes de cycles de vie, la jeunesse est l'intervalle où se jouent l'autonomisation et l'intégration professionnelle et sociale. Des bornes, c'est-à-dire des âges attachés à des événements qui sanctionnent des passages, définissent, d'un côté, l'entrée dans la jeunesse et, de l'autre, le passage à l'âge adulte. On comprend que, dans une telle optique (Galland, 1997), on puisse insister sur l'allongement de la jeunesse comme un fait social, dont les transformations les plus visibles, d'un contexte à un autre, sont d'abord une affaire de décalage. Question de temps, mais aussi de modalités "d'insertion", puisque l'étalement de la jeunesse s'explique par " (la) contrainte de mobilité et d'intégration d'un côté, et (le) blocage et (la) fermeture des issues et des accès connus de l'autre » (Marie, 1997, p. 11). Il est alors important d'appréhender cette "double injonction "(Castel, 1995), non seulement dans les désynchronisations qu'elle induit, mais également dans les nouveaux arbitrages et modes de vie qu'elle suscite ou rend visibles, pour chacun des trois passages (sortie du système scolaire, entrée dans la vie active, mariage et décohabitation) ou plutôt des trois biographies (scolaire, professionnelle, familiale).

Le projet éducatif : allongement de la scolarité et retard de l'entrée dans la vie active

Des années 1960 aux années 1990, les statistiques nationales'reflètent les changements du paysage éducatif. La généralisation de l'accès à l'éducation et l'allongement de la scolarité sont deux changements significatifs entre la génération des parents et celle de leurs enfants. Alors qu'un parent sur deux est analphabète', tous les enfants ont été scolarisés : huit d'entre eux savent lire et écrire, vingt-trois ont achevé le cycle primaire et autant le secondaire. Troisième observation notable, à l'allongement de la scolarisation s'ajoute également la diminution des écarts entre les filles et les garçons. Dans notre population, les plus jeunes femmes ont accès à l'enseignement supérieur dans les mêmes proportions que leurs frères. Par l'accès, la durée et l'orientation, la situation éducative des jeunes d'aujourd'hui n'a donc pratiquement rien à voir avec celle de leurs parents (Fargues, 1995).

6 Au sein des fratries, la distribution des jeunes, par niveau d'éducation et selon les âges, indique que la sortie du système scolaire s'apparente, pour les aînés, à un abandon (essentiellement pendant et, plus rarement, à la fin du primaire), alors que les plus jeunes sortent diplômés du secondaire technique ou général. Symétriquement, l'ensemble des jeunes de niveau universitaire est âgé de moins de 22 ans. Si la déscolarisation des aînés (garçons) s'est faite plus progressivement que pour leur père, elle s'est aussi accompagnée d'une mise en apprentissage dans l'économie informelle. On observe ainsi que, dans de nombreux cas, scolarisation et mise au travail ne sont pas antinomiques (Zibani, 1994). Au contraire, la déscolarisation des jeunes femmes a très rarement signifié leur entrée sur le marché du travail (un cas). Lorsque la déscolarisation est devenue "inévitable», leurs mères, moteurs de l'économie domestique, peu présentes sur le marché du travail, ont "trouvé à l'extérieur des revenus supplémentaires » (Fatîha, 53 ans), par le biais d'activités de couture ou de petits commerces, qu'elles ne qualifient cependant pas d'emploi. 
7 La déscolarisation est donc avant tout une affaire de budget, elle intervient en effet en dernier recours. L'investissement matériel et symbolique des familles dans le système éducatif reflète à la fois les sacrifices qu'un tel arbitrage impose et les attentes en termes de statut social et professionnel que suscite le fait d'être diplômé. Les représentations du marché du travail" souvent très pessimistes n'estompent pas pour autant les espérances et la confiance des jeunes dans le système scolaire. Le choix de l'éducation est associé à l'idée d'un «bon travail» (Muhammad, 24 ans), mais avant tout à l'espoir d'une mobilité sociale liée au fait d'être éduqué, ce que n'étaient pas les parents.

Biographie professionnelle : chômage et instabilité

De fait, les attentes des parents et des enfants sont d'autant plus importantes que le choix de l'éducation se fait au prix d'efforts humains et d'investissements matériels. Or l'entrée dans la vie active est non seulement retardée (près de 10 ans de différence) du fait d'une scolarisation plus longue, mais elle se caractérise désormais par un passage presque automatique par le chômage et reflète en termes de biographie une très grande instabilité professionnelle et l'inexistence de projet professionnel.

Le chômage comme premier statut d'actif" est une donnée fréquente des biographies professionnelles. En 1996/1997, 160000 jeunes ont été diplômés du supérieur, des universités et des instituts techniques (CAPMAS, 1998). Tous niveaux éducatifs confondus, les nouveaux entrants sur le marché du travail forment chaque année un groupe de 450000 à 500000 chercheurs d'emploi (Enquête emploi par sondage, 1998). L'incapacité du marché du travail à absorber ces nouveaux demandeurs d'emploi se traduit par un taux de chômage croissant, particulièrement parmi les jeunes. En 1996, sur 1459995 chômeurs recensés", $5 \%$ ont déjà travaillé, $95 \%$ sont des nouveaux chômeurs. Parmi ces nouveaux demandeurs d'emploi, tous âgés de moins de 35 ans, 69 $\%$ ont entre 20 et 30 ans. Qu'ils soient nouveaux chômeurs ou qu'ils aient déjà travaillé", $21 \%$ des jeunes actifs âgés de 20 à 30 ans sont au chômage (respectivement, 36 \% des jeunes femmes et $17 \%$ des jeunes hommes)".

10 Les explications mobilisées sont d'ordre économique, renvoyant aux déséquilibres entre la population active et le marché du travail. Le chômage s'explique en premier lieu par l'augmentation de la population active et l'insuffisante création d'emplois. La plus grande participation des femmes au marché du travails et l'importance de la population jeune" engendrent un nombre de demandeurs d'emploi croissant. Compte tenu du taux de croissance actuel de la population active (2,6\%), 600000 à 700000 emplois devraient être créés annuellement pour absorber les nouveaux entrants et les chômeurs (Fergany, 1998)". En second lieu, le chômage, notamment des jeunes, et le sous emploi s'expliquent par les divergences entre la structure de la population active et celle du marché du travail. Plus centrée sur les résultats et performances macroéconomiques, la deuxième explication se réfère plus spécifiquement à la structure du marché du travail et l'évolution des salaires. Elle mobilise la dynamique de création d'emplois (politiques publiques, ajustement structurel et privatisation) autour d'une périodisation de l'état du marché du travail en trois moments (les années 1960, années du surplus de main-d'œuvre ; les années 1975-1985, période d'ouverture; et les années 1985-1995, décennie du chômage ouvert). Dans ce sens, les travaux du Bureau international du travail mettent en évidence les relations entre la diminution des salaires réels (deux tiers du salaire base 1985 en 1995), la répartition de la population 
active occupée, la productivité des différents secteurs et les politiques de l'emploi (Radwan, 1997).

11 L'insertion problématique ou l'allongement de la transition professionnelle reflète ainsi la rareté des débouchés et génère de nouvelles modalités d'accès à l'emploi. La mobilisation des réseaux (professionnels, amicaux, parentaux ou confessionnels) reste, d'une génération à l'autre, une constante des biographies professionnelles. Cependant, du fait de la structure du marché du travail et de l'insatisfaction des attentes générées par le diplôme (occupational reality shock, H. T. Foster, 2000), les réseaux sont devenus un outil majeur dans les arbitrages professionnels. Alors que les itinéraires professionnels des parents traduisent soit une plus grande stabilité professionnelle, soit une mobilité inscrite dans un projet", leurs enfants sont plus instables. Les jeunes (principalement les jeunes hommes) changent très régulièrement d'emploi (en moyenne dans notre échantillon, cinq fois par an). Ces tribulations professionnelles ne s'apparentent pas à une accumulation de compétences ; au contraire, passé un premier recyclage de leur savoirfaire, de nombreux jeunes ne cherchent plus à se stabiliser professionnellement dans un secteur donné. Cet éparpillement suggère une multiplication d'activités supposant des qualifications et des statuts différents. On en distingue deux formes, la pluriactivité (deux, parfois trois emplois par jour) et le nomadisme professionnel (quatre à sept emplois par an), entre lesquelles existe un continuum de situations professionnelles où les deux cas de figure se conjuguent et coexistent dans un itinéraire marqué par "l'empilement d'activités et de compétences » (Roulleau-Berger, 1999).

L'entrée dans la vie active et le maintien sur le marché du travail ne sont donc possibles qu'au prix de "stratégies" (mobilisation de réseaux, migration, pluriactivité, sousemploi). Dans les biographies professionnelles des jeunes, la stabilité (dans l'emploi, dans un statut ou un secteur) n'est plus aussi évidente que pour la génération des parents: elle se combine toujours ou presque à une très forte révocabilité des engagements des jeunes. L'existence de situations professionnelles stables dans des biographies marquées par l'instabilité s'explique par la "réversibilité » même des positions et des représentations du monde de l'emploi. Perçues communément (par les médias, les familles, les politiques publiques) comme des carrières de précarité, les biographies professionnelles se construisent autour d'expériences professionnelles qui prennent le sens d'un ajustement, d'un compromis toujours négociable et négocié. Ainsi, certains jeunes - au-delà du statut éducatif, c'est surtout une affaire d'âge - ne cherchent pas d'emploi stable, parce qu'un emploi stable ou garanti, « ça n'existe plus » (Ashraf, 23 ans), et que les avantages (assurance sociale, contrat, par exemple) ne compensent les inconvénients (statuts souvent dévalorisés, « salaire dérisoire » (Imân, 23 ans), irrégularité du paiement du salaire) que dans la mesure ou une autre activité vient se greffer à la journée de travail. Cette dynamique sous-tend des représentations du marché du travail, où la situation professionnelle est avant tout affaire de conciliation. Il faut à ce niveau distinguer un «bon travail» d'un «bon compromis professionnel». Un bon travail est avant tout un «bon emploi », qui est affaire de stabilité (shughl madmûn), de salaire équitable, de conditions de travail supportables (essentiellement, la distance séparant le domicile du lieu de travail, l'environnement professionnel et le type d'emploi - décent - pour les jeunes femmes). Devant l'impossibilité de trouver "la perle rare » (Sâmya, 22 ans), il s'agit, en jonglant entre différentes activités - il n'est plus tant question d'emploi -, d'atteindre un équilibre supportable. La diversité des arbitrages, l'instrumentalisation de réseaux, la mobilité (notamment spatiale) des jeunes construisent leurs expériences professionnelles autour 
de compétences sans cesse réactualisées. Mais l'enjeu de ces ajustements n'en est pas pour autant strictement professionnel et ne saurait se comprendre par les seules représentations des mondes de l'emploi.

En effet, cette hyper mobilité traduit de nouveaux rapports à l'emploi des jeunes que définissent des « dosages » différents de projets professionnels et matrimoniaux. On en distingue trois formes. Rares sont les cas où la formulation d'un projet professionnel oriente les arbitrages en termes d'emploi et met en avant l'idée de carrière. Seuls deux garçons et une fille, sur les 60 jeunes, formulent un projet et anticipent leur avenir professionnel". La plupart des jeunes naviguent entre désillusion professionnelle et "utopie matrimoniale» (Ashraf, 29 ans). Face au déclassement structurel de leurs qualifications et à l'insatisfaction de leurs attentes (soit parce qu'ils ne trouvent pas d'emploi correspondant à leur formation, soit parce qu'au-delà de la définition de l'emploi, leurs qualifications ne sont pas mobilisées), l'instabilité professionnelle signifie une sorte de démission des jeunes et leur incapacité à remplir «leur contrat » (Wâ'il, 24 ans), celui d'une quête de l'autonomie qu'ils n'ont plus les moyens d'atteindre. Il n'y a plus de projections, la jeunesse devient un moratoire. Enfin, l'hyperactivité des jeunes traduit aussi l'investissement matériel que suscite la préparation du mariage. La dispersion professionnelle est alors justifiée par la nécessaire accumulation financière et l'impossibilité structurelle à "satisfaire ses propres compétences» (Mahmûd, 26 ans). Entre la course au mariage et l'illusoire réalisation professionnelle, certains jeunes choisissent d'investir dans leur mariage plutôt que dans une hypothétique carrière sur le marché du travail.

Mariage et décohabitation : utopie ou épreuve

Le mariage est au cœur des récits professionnels. Qu'il soit le moteur de l'itinéraire ou qu'au contraire les jeunes se désengagent de la «course au mariage» (Ahmad, 25 ans), son importance se construit dans la référence qu'il constitue ${ }^{w}$. La centralité du thème dans les discours des jeunes fait écho à la récurrence que constitue cette même question, notamment dans la presse, où l'accent est mis sur le recul de l'âge au mariage comme symptôme d'une crise économique et « morale ».

Revenir sur le projet matrimonial des parents n'est pas chose aisée : comme pour leurs biographies professionnelles, les vingt ou trente ans qui se sont écoulés depuis ne permettent pas de comparer strictement ces reconstitutions aux récits des jeunes d'aujourd'hui. Outre que l'on n'atteint pas le même degré de détail, on est conscient du biais de réinterprétation des souvenirs tant professionnels que matrimoniaux (Couppié, Demazière, 1995). Cependant, on peut considérer que, d'une génération à l'autre, les modifications les plus visibles, celles de l'allongement de la période d'attente et des préparations, construisent de nouvelles modalités amoureuses (sens et formes des engagements) et de nouvelles relations familiales.

16 Les difficultés inhérentes au mariage se traduisent d'abord dans le recul de l'âge moyen au premier mariage. L'importance en est sujette à des observations différentes, selon qu'il s'agit des zones rurales ou urbaines, des jeunes hommes ou des jeunes femmes". Dans notre population, le mariage est différé d'environ 10 ans : alors que les parents se sont mariés en moyenne à 22 ans ( 24 ans pour les hommes, 19 ans pour les femmes), au moment de nos entretiens (et jusqu'à la fin de notre terrain), seulement deux jeunes sont mariés (ils ont 28 et 29 ans), quatre autres sont fiancés ; plus d'un jeune sur deux a, par contre, déjà été fiancé au moins une fois. Si la question de la durée renvoie notamment à l'instabilité professionnelle et plus largement à des questions de budget 
(Singerman, 1997), les modalités selon lesquelles se joue le projet matrimonial ont également changé.

Du temps des parents, tous les mariages de notre population ont été arrangés par les familles ; ils s'inscrivaient en outre pour le jeune homme dans un projet professionnel : s'établir à son compte ou migrer. La migration vers les pays du Golfe avait généralement lieu après les fiançailles ou après la naissance des premiers enfants (Hoodfar, 1999) et permettait, à la fois, de financer le mariage (dont le coût était moindre, notamment la dot, mahr ${ }^{2}$ ) et d'entretenir le foyer. Pour la génération des enfants, les possibilités migratoires se sont restreintes au territoire national, sur la base de réseaux en outre plus difficiles à mobiliser. Ainsi, l'épargne nécessaire au financement du mariage en retarde la réalisation. Elle se conjugue cependant à d'autres raisons. Cette situation a d'abord à voir avec l'éducation des jeunes femmes : lorsque ces dernières poursuivent des études, le mariage a lieu après l'obtention du diplôme. En outre, la dot demandée par les parents est plus élevée, au motif que la jeune femme est diplômée. Ensuite, la difficulté à trouver un logement retarde encore la décohabitation au-delà même de l'objectif du mariage. Les couples mariés, lorsqu'ils n'ont pas la possibilité de déménager, résident chez les parents du mari, dans une extension du domicile ou dans une pièce qui leur est réservée. Cependant, lorsque cet arrangement est possible, il ne concerne que les aînés. $\mathrm{Si}$, d'une manière générale, le mariage demeure le premier transfert financier intergénérationnel et si les jeunes participent de manière croissante au financement du mariage (les jeunes femmes à un moindre degré), la somme à réunir pour financer le mariage et l'installation du couple dépasse les possibilités offertes par le marché du travail ou les possibilités d'emprunt associatif informel (gamiiyyât).

18 Ainsi observe-t-on que, de 20 à 30 ans, de nombreux jeunes sont « en attente » (Amal, 24 ans). Mais l'impossible ou la difficile réalisation du cahier des charges à la fois professionnel et matrimonial, donne cependant à voir de nouvelles formes d'expériences de la jeunesse. La multitude des engagements - avant le mariage, les fiançailles peuvent avoir été rompues une à deux fois - reflète en partie l'ampleur des négociations entre les familles, mais traduit également de nouvelles aspirations des jeunes et l'usage qu'ils font de leur jeunesse. Le plus souvent, les négociations qui ont à voir directement avec le mariage ne laissent officiellement que peu de place aux jeunes. On observe ainsi que les fiançailles jouent un rôle de monitoring, de contrôle de moralité de la part des parents, et sont simultanément une période que les jeunes, même lorsqu'ils «travaillent pour se marier» (cAliyya, 27 ans), envisagent comme les premiers moments d'autonomie. De nombreuses jeunes femmes apprécient la «marge de manœuvre » (Amira, 24 ans) dont elles disposent. Présentes sur le marché du travail ou à l'université, elles bénéficient déjà d'une autonomie plus grande que celle de leur mère. Le fait d'être fiancée confère en outre un statut où les possibilités de sorties sont plus grandes, même chaperonnées. Les contraintes morales et sociales et les rebondissements qu'impliquent les négociations familiales (Hoodfar, 1999) n'épuisent ainsi pas toujours les ressources et l'ingéniosité des jeunes. Les lieux - du travail au jardin public - et les moments de rencontre s'enchevêtrent et se multiplient dans des usages et des stratégies qui diffèrent du modèle parental. De manière moins visible, l'implication des plus jeunes s'illustre dans la «résistance» ou la négociation des décisions qui les concernent, au sein de la famille ou du futur couple (Nawar, Lloyd, 
Ibrahim, 1995). L'importance de ces négociations - qui sont aussi des compromis - se mesure dans les limites de la dépendance des jeunes du foyer familial.

Le Chômeur et le prétendant : les nouvelles « figures » jeunes

Les désynchronisations des biographies professionnelles et familiales diffèrent les passages qui sanctionnent des changements de statut et rendent ainsi plus visibles certaines populations jeunes. Alors que les formes tout autant que les âges et les expériences de la jeunesse se multiplient, l'image dominante est celle d'une génération en crise. Image qui se construit en référence tant au contexte présent (crise de l'emploi, inadéquation du système éducatif au marché du travail, crise du logement...) qu'à un sens passé - presque obsolète? - de la jeunesse (perte des valeurs morales, désœuvrement, irresponsabilité des jeunes de la «nouvelle » génération). Qu'ils soient traités sur le thème de la prévention (les adolescents) ou de l'intervention (les diplômés, les chômeurs), ils se construisent autour d'un sens de la jeunesse qui ne serait plus le moment où se joue et se résout l'intégration professionnelle et sociale, mais celui de la quête désespérée et souvent illusoire de l'autonomie. Précisément, parce que l'instabilité professionnelle serait synonyme - voire cause - de l'instabilité sociale, les représentations se cristallisent dans l'idée de la crise, de l'impuissance des jeunes et de leurs familles et du déclassement de leurs compétences tant professionnelles que sociales. Les nouvelles figures jeunes construites en écho aux maux de la jeunesse sont ainsi autant de symptômes traduisant la nécessité d'une intervention en direction de la jeunesse et catalysant l'expression d'une crise sociale plus large, où il en va de l'avenir de la société face au malaise de sa jeunesse.

Les nouveaux âges de la jeunesse : n'est pas jeune qui veut

Alors même que les travaux français et plus largement européens (Mauger, 1994a, 1994b) ont mis l'accent sur la nécessaire critique de la prénotion de jeunesse, les recherches sur les jeunes en Égypte appréhendent une jeunesse d'emblée fortement catégorisée et polarisée. « Être jeune » renvoie en effet, selon les besoins du législateur, $\mathrm{du}$ statisticien ou du chercheur, à des attributs qui sont autant affaire d'âge que de statut. Les multiples usages de la jeunesse produisent ainsi une constellation de définitions et de découpages (Shafey, 1998), où la légitimité (on est jeune au titre d'un âge ou d'une situation) se construit aussi autour de la plus grande visibilité de certains groupes, au premier rang desquels les adolescents, les jeunes diplômés, les chômeurs ou les prétendants.

21 L'intérêt manifesté pour les plus jeunes des jeunes, les plus précaires ou les plus diplômés renvoie aux ruptures et aux dysfonctionnements d'un modèle où l'intégration des jeunes et leur participation étaient affaire d'enchaînements chronologiques d'accès et de situations. Dans les années 1990, alors que les jeunes sont à la fois plus nombreux et mieux qualifiés, le contexte économique et social changeant (plan d'ajustement structurel, retour de la main-d'œuvre émigrée dans les pays du Golfe, réduction de l'embauche des diplômés dans le secteur public) ne permet plus d'absorber les nouveaux demandeurs d'emploi. Pour n'être pas un phénomène nouveau, le chômage des jeunes, en se généralisant (Fargues, 1998), devient un problème social précisément parce que les jeunes diplômés "alimentent la file des morts " (lire chômeurs, al-Wafd, 14/11/98). En se saisissant des «maux de la jeunesse » (mashâkil al-shabâb), les médias contribuent ainsi à l'émergence de ces nouvelles catégories, celles de l'expertise, qui constituent aussi les nouvelles cibles des politiques publiques. La perméabilité des catégories administratives et des usages médiatiques de la jeunesse a pour effet de 
produire l'image d'une génération en crise, dont les déviances sont affaire de drogue, de violence, de perte de valeurs à la fois morales et religieuses ${ }^{2}$.

Premières « victimes des nouvelles règles du jeu » (Salwa, 27 ans), les jeunes sont aussi les premiers bénéficiaires des mesures prises dans le cadre de la dimension sociale de l'ajustement. La multiplication des projets en direction des jeunes diplômés (khirrîgîn), les créations d'emplois pour les jeunes, la distribution de terres bonifiées ou l'accès à des micro-crédits pour financer, qui ont pour objectif le développement de l'autoemploi et des petites entreprises, trouvent un écho important dans la presse. Toute personne diplômée depuis 1985 peut monter un dossier et prétendre à un crédit ou à une aide selon les projets (al-Ahrâr, 23/11/1998). Ainsi, les projets-jeunes en matière d'emploi - il en va de même en matière de logement - définissent des populations jeunes, de plus en plus «âgées ». Par exemple, dans le cadre du projet Moubarak, lancé en 1996, 50000 habitations à prix modérés sont destinées aux jeunes âgés de 29 à 35 ans, la priorité étant donnée aux jeunes mariés. Autre projet, autres âges: les logements de l'avenir (iskân al-mustaqbal) à destination des "personnes jeunes » ou « à faible revenu » sont construits dans les villes nouvelles. Les " personnes jeunes » sont âgées de 30 à 45 ans, « âge du mariage » en Égypte (Florin, 1999, p. 254).

Alors que la construction de catégories scientifiques, le plus souvent issues des définitions internationales que l'on voudrait opérationnelles, a pour effet de lisser et d'homogénéiser la jeunesse autour de la tranche 15-24 ans (al-Tawila, 2000), l'intervention en direction de la jeunesse ou de certaines jeunesses en Égypte (qu'en est-il des jeunes non diplômés, des jeunes ruraux... ?) contribue à l'éclatement et à la dilution d'un objet déjà fragmenté. Dans cette double tension, l'image des jeunes se construit autour de figures jeunes, celles du chômeur ou du prétendant, dont l'apparition doit au contexte autant démographique qu'économique et social et dont la visibilité, pour être statistique, renvoie également au sens attribué à la jeunesse. Ainsi, tant au niveau du sens commun, des médias, des catégories de l'expertise qu'à celui des politiques publiques, le sens de la jeunesse est celui d'une "chronologie d'insertions", dont l'issue, le passage à l'âge adulte, est validée par le mariage. De la communauté aux experts, la mesure de l'autonomie (corollaire de l'âge adulte) renvoie donc à la réalisation d'un modèle prescriptif, dont le mariage est la norme (Singerman, 1997, Sherif, 1999). L'incapacité à satisfaire ces modalités professionnelles et matrimoniales diffère le passage à l'âge adulte ; la responsabilité en incombe tout à la fois aux jeunes et à la société et questionne le sens de l'engagement et de la participation des jeunes à la société.

Des « figures » jeunes

24 La jeunesse, dont les médias façonnent pour partie l'image, s'incarne dans la figure du chômeur ou du prétendant. Dans un cas comme dans l'autre, ces représentations illustrent les écueils de la jeunesse. Elles se construisent autour des situations que vivent les jeunes, en référence aux nouvelles orientations politiques les ciblant. Le caractère « exemplaire » de ces représentations induit une attention singulière sur les individus, au-delà, de leur condition: il est ainsi question du chômeur plus systématiquement que du chômage, du prétendant plus que du mariage.

L'accent, mis sur les individus plus que sur les situations qu'ils vivent, dessine un profil dont les attributs synthétisent l'ensemble des projections sur la jeunesse. La masse anonyme des jeunes diplômés est assimilée aux portraits qu'en font les médias. Cette image se construit unilatéralement et rend compte de l'expérience collective du 
chômage des jeunes. Le problème du chômage est alors une question d'entrée dans la vie active - perçue dans les seuls termes de "l'insertion professionnelle »- et alimente ainsi le débat sur les relations entre marché du travail et système éducatif".

$\mathrm{Au}$ premier rang des thèmes abordés, figure la question de la responsabilité et des efforts entrepris pour « sauver la jeunesse » (al-Akhbâr, 15/07/99). Les jeunes chômeurs, bénéficiaires des politiques publiques, sont aussi désignés comme partiellement responsables de leur insertion professionnelle, même si cette dernière est avant tout structurée par les possibilités qu'offre le contexte - en tant que moment spécifique - et qu'elle renvoie aux politiques plus qu'aux pratiques des acteurs. Le désœuvrement et l'usage du temps libre apparaissent alors comme des thèmes centraux dans la problématique de l'emploi et du chômage, même s'ils renvoient d'abord aux polémiques sur les centres de jeunesse $\mathrm{e}^{*}$ (markaz al-shabâb). Outre la représentation stéréotypée d'un jeune au chômage, qui le met en scène assis au café, les articles s'accordent le plus généralement pour s'interroger sur les aspirations professionnelles des jeunes. Pourquoi ces derniers continuent-ils à chercher des emplois dans le secteur public ou gouvernemental, s'interroge le ministre de la Main-d'œuvre, Ahmad al'Amâwî (al-Ahrâm, 19/11/99), alors que le secteur privé leur offre des salaires plus confortables et que les politiques publiques, par le biais du micro-crédit, permettent à de nombreux jeunes de monter un dossier de prêt et de créer leurs propres emplois en s'équipant ou de bénéficier de terres bonifiées? Pourquoi demeurent-ils dans des emplois à «bakchich » (al-Wafd, 15/01/99), alors qu'ils pourraient postuler aux offres du Fonds social pour le développement (FSD) ? Pourquoi, enfin, alors que l'État œuvre pour sa jeunesse, les jeunes eux-mêmes baissent-ils les bras (al-Ahrâm al-iqtisâdî, 05/07/99 ; Rûz al-Yûsuf, 18/12/99) ? Le point commun de ces interrogations réside dans l'incompréhension des aspirations des jeunes. Pour autant qu'ils puissent avoir accès aux politiques qui les ciblent, les jeunes désespèrent de travailler dans certains projets où, non seulement leurs compétences et qualifications professionnelles ne sont pas mobilisées, mais où leur statut est en outre dévalorisé.

Le discours actuel sur les jeunes - chômeurs - renvoient à la mobilisation d'un sens de l'engagement ou du civisme, en termes, par exemple, d'environnement, de nettoyage des rues ou de jardinage, où la définition même du sens de l'engagement est unilatérale. Les médias atténuent parfois cette première lecture par l'analyse critique des projets proposés aux jeunes, mais la conclusion reste unanime, les jeunes sont partiellement responsables de leur situation. Il ne tient qu'à eux, puisque tous les efforts sont mis de leur côté, de réussir leur insertion professionnelle.

Dans les médias, la situation professionnelle et plus largement sociale des jeunes se construit dans la référence à la jeunesse de leurs parents, elle oscille entre une position de victime (qui renvoie au contexte) et une part de responsabilité. De la même manière, la mise en scène du parcours du prétendant se construit aussi dans la dénonciation des comportements « déviants » de la jeunesse. Qu'il s'agisse du mariage 'urfi', du célibat ou du divorce, le malaise des jeunes, en étant traité comme une affaire de mœurs et de perte de valeurs, met en exergue l'avenir de la société et la responsabilité familiale. La transmission des valeurs familiales et l'harmonie familiale sont, de fait, au centre de la crise que traverse la famille en tant qu'institution: modèle de socialisation et de réalisation de soi, la famille ne jouerait plus son rôle, titrent les médias. Dans notre population, les médiations et les négociations au sein de la famille montrent à quel point sont intériorisées les normes parentales, intériorisation d'autant plus forte que la 
situation du jeune (situation sociale ou/et professionnelle) dépend de réseaux familiaux. Les changements qui traversent la structure familiale sont parfois assez difficiles à appréhender. Cependant, l'attitude des jeunes, notamment de ceux qui sont fiancés, envers toute forme de changement (de l'organisation familiale, la planification $\mathrm{du}$ foyer, aux questions de prise de décision et de gestion du budget) semblent être affaire d'âge, d'éducation et plus largement, de position au sein de la famille. On entend par là que les aînés ont été plus enclins à reproduire le "modèle" parental. Par ailleurs, l'ancrage dans des réseaux (familiaux ou confessionnels) module encore ces premiers résultats. Il semble ainsi que les décisions ayant trait au mariage (choix du conjoint, nombre d'enfants, travail de la femme, par exemple) renvoient à de nouvelles contraintes ou à de moindres possibilités, mais également à de nouveaux choix.

L'entreprise familiale que représente le mariage - de l'organisation matérielle aux négociations entre les familles - est ainsi le plus souvent vécue comme une épreuve, " un film dont on est tous les figurants » ('Amr, 27 ans), où les délais (de 5 à 7 ans) sont aussi une affaire de contrôle de la part des parents et de négociations de la part des jeunes.

Conclusion

«Le jeune musulman qui veut se marier - afin de ne pas commettre le péché - doit économiser le total du montant de sa paye pendant vingt ans pour pouvoir payer la dot; il a besoin de vingt autres années pour pouvoir payer un acompte pour un appartement - et cela si les prix n'augmentent pas. Sinon, il passera sa vie à économiser le montant de ses funérailles " (Hishâm Abû al-Dhahab, 3eannée de commerce, al-Ahrâr, 5 octobre 1981) ${ }^{\text {}}$. Pour n'être pas récents, mais plutôt récurrents ('Abd al-cAzîz, 1977, Fouad, 1986), les problèmes des jeunes prennent depuis quelques années une place centrale dans les discours et débats sur la jeunesse. La question du malaise de la jeunesse, apparentée d'abord aux échecs du système éducatif et à la responsabilité de la famille, se construit désormais dans l'espace très médiatisé des politiques publiques. Depuis le début des années quatre-vingt-dix (coïncidant ainsi avec les politiques d'ajustement structurel et leur volet social), l'impossible reproduction du modèle familial (embrassant par-là même les questions professionnelles comme « support » du projet matrimonial) donne à voir de nouveaux modes de vie et de nouveaux âges et/ou figures de la jeunesse. Cependant, si ces arbitrages se construisent pour partie dans l'impossibilité à réaliser un "contrat " qui mène de la jeunesse à l'âge adulte, ils amènent à interroger « la jeunesse ", non plus dans les seuls termes des âges, mais aussi dans les différentes formes d'expériences et le sens que les jeunes y attachent.

En effet, en limitant la question de "la jeunesse " aux fluctuations des bornes qui l'enchâssent, on tend à produire une image uniforme et "linéaire » de la jeunesse, entendue comme une biographie unique. En privilégiant la reconstitution biographique, on interroge moins l'enchaînement des événements que les diverses formes et sens qu'ils prennent tant pour les jeunes qu'au regard de l'air du temps, celui d'un impossible passage à l'âge adulte. Au-delà d'une chronologie d'insertions, la quête de l'autonomie renvoie essentiellement aux nouveaux modes de vie (par les arbitrages, les représentations et les expériences de ces «jeunes adultes») qui se tissent autour d'un impossible « contrat à remplir ». 


\section{BIBLIOGRAPHIE}

‘Abd Al-cAzîz Muhammad, 1977, Les problèmes de la jeunesse, leur orientation, leurs aspirations (en arabe), Le Caire, machad al-takhtît al-qawmî, 121 p.

Al-Tawila Sahar, 2000, Youth in the Population Agenda: Concepts and Methodologies, Le Caire, Population Council (West Asia and North Africa), 26 p. - (Collection « MEAWARDS Regional Papers ", $\left.\mathrm{n}^{\circ} 44\right)$.

Beaud Stéphane, 1996, « Un ouvrier, fils d'immigré "pris” dans la crise : rupture

biographique et configuration familiale ", Genèses, Trajectoire, Septembre 1996, n² 24, p. 5-32.

Castel Robert, Les métamorphoses de la question sociale, une chronique du salariat, Paris, Fayard, Collection « L'espace du politique », 1995, $490 \mathrm{p}$.

Couppié Thomas, Demazière Didier, 1995, « Se souvenir de son passé professionnel :

appel à la mémoire dans les enquêtes rétrospectives et construction sociale des données ", Bulletin de méthodologie sociologique, décembre 1995, nº 49, p. 23-49.

Courbage Youssef, 2000, « The demographic inflection of the Southern Mediterranean: Reasons for optimism ", in Meijer, Roel (éd.), Alienation or Integration of Arab Youth. Between Family, Sate and the Street, Richmond, Curzon Press, p. 27-43.

Courbage Youssef, 1999, Issue of fertility transition in the Middle East and North Africa, The Economic Research Forum for Arab Countries (ERF), Le Caire, Collection « Working paper », n 9903, 25 p.

Courbage Youssef, 1995, « Fertility transition in the Mashriq and the Maghrib: Education, Emigration, and the Diffusion of Ideas ", in Makhlouf Obermeyer Carla (éd.), Family, Gender and Population in the Middle East. Policies in Context, Le Caire, The American University in Cairo Press, p. 80-104.

Demazière Didier, Dubar Claude, 1997, Analyser les entretiens biographiques, l'exemple de récits d'insertion, Paris, Nathan, Collection « Essais et Recherches », 350 p.

Dubet François, 1994, Sociologie de l'expérience, Paris, Seuil, Collection « La couleur des idées », 272 p.

Evans Karen, Furlong Andy, 2000, « Niches, transitions, trajectoire... De quelques théories et représentations des passages de la jeunesse », Lien social et politique - RIAC, Voir les jeunes autrement, $n^{\circ} 43$, Printemps 2000, p. 41-48.

Fargues Philippe, 1998, « La montée du chômage en Égypte (1960-1995) », Égypte/Monde arabe, $1^{\text {er }}$ trimestre $1998, \mathrm{n}^{\circ} 33, \mathrm{p} .147-179$.

Fargues Philippe, 1995, « Changing hierarchies of gender and generation in the Arab world », in Makhlouf Obermeyer Carla (éd.), op. cit., p. 179-198.

Fergany Nader, 1998, Dynamics of Employment Creation and Destruction. Egypt, 1990-1995, Le Caire, Almishkat Research Center, notes $n^{\circ} 11,20 \mathrm{p}$.

Florin Bénédicte, 2000, Itinéraires citadins au Caire : Mobilité et territoires dans une métropole du monde arabe, Thèse de Doctorat, URBAMA, Université François Rabelais - Tours, sous la direction de Pierre Signoles, 561 p. 
Foster Howard T., Howard Marla, 2000, « Job Expectations of Moroccan Youth », The New Generations South of the Mediterranean. Changes, Challenges and Opportunities, Institut Universitaire Européen - Florence, 22-26 mars 2000 (non publié).

Fu'âd Ahmed, 1986, Crise de la jeunesse et soucis égyptiens (en arabe), Le Caire, Dâr al-ictisâm, 238 p.

Galland Olivier, 1997, Sociologie de la jeunesse, Paris, Armand Colin, Collection « U », Masson, 247 p.

Hoodfar Homa, 1999, Between Marriage and the Market - Intimate Politics and Survival in Cairo, Le Caire, The American University in Cairo Press, 302 p.

Hughes E. C., 1996, Le regard sociologique, Essais choisis, Paris, Édition EHESS, 344 p.

Lautier Bruno, 1995, «Cycle de vie, trajectoire professionnelle et stratégies familiales. Quelques réflexions méthodologiques à partir de travaux latino-américains ", dans Cabannes Robert, Copans Jean, Selim Monique (éds), Salariés et entreprises dans les pays du Sud. Contribution à une anthropologie politique, Paris, Karthala - ORSTOM, Collection « Homme et Société », p. 335-358.

Marie Alain (éd.), 1997, L'Afrique des individus, Paris, Karthala, Collection « Hommes et Sociétés », $438 \mathrm{p}$.

Mauger Gérard, Bendit René, Von Wolffersdorff Christian, 1994a, Jeunesses et sociétés. Perspectives de recherche en France et en Allemagne, Paris, Armand Colin, Collection « Bibliothèque européenne des sciences de l'éducation », 355 p.

Mauger Gérard, 1994b, Les jeunes en France. État des recherches, Paris, La Documentation française, 1994, 295 p.

Meijer Roel (éd.), 2000, Alienation or Integration of Arab Youth. Between Family, State and the Street, Richmond, Curzon Press, $223 \mathrm{p}$.

Nawar Leila, Lloyd Cynthia B., Ibrahim Barbara, 1995, « Women's autonomy and gender roles in Egyptian families », in Makhlouf Obermeyer Carla (éd.), op. cit., p. 147-178.

Radwan Samir, 1997, Job Creation and Poverty Alleviation in Egypt: Strategy and Programmes, Genève, BIT, n. p.

Roulleau-Berger Laurence, 1999, Le travail en friche, les mondes de la petite production urbaine, La Tour d'Aigues, Éditions de l'Aube, Collection « Essai », 245 p.

Shafey Halla, 1998, Adolescence and State Policy in Egypt, Le Caire, The Population Council, 85 p. Sherif Bahira, 1999, « The prayer of a married men is equal to seventy prayers of a single man: the central role of marriage among upper-middle class Muslim Egyptian », Journal of Family Issues, September 1999, vol. 20, nº 5, p. 617-632.

Singerman Diane, 1997, Avenue of participation. Family, Politics and Networks in Urban Quarters of Cairo, Le Caire, The American University in Cairo Press, 335 p.

Yousef Tarik, 2000, «Demography, Labor Markets and the Politics of Job Creation in the $21^{\text {st }}$ Century », The New Generations..., op. cit.

Zibani Nadia, 1994, « Le travail des enfants en Égypte et ses rapports avec la scolarisation Esquisse d'évolution », Égypte/Monde arabe, 2 et 3etrimestre 1994, n 18-19, p. 135-167. 


\section{NOTES}

1.Médias, politiques publiques et productions savantes sont alors autant d'éléments à considérer.

2.Les quartiers choisis sont Izbat al-Ward (Macâdî), Machad Nâsir (Shubra) et Warrâq alhadar (Imbâba).

3.Vingt-cinq entretiens ont été réalisés avec les parents, soixante avec les enfants.

4.On entend par-là les âges qui mobilisent l'intérêt et sont porteurs du monopole de la jeunesse et la récurrence ou l'apparition de thèmes identifiés ou présentés comme typiquement « jeunes».

5.Ou double bind, " pris entre l'injonction de travailler et l'impossibilité de travailler selon les formes prescrites » (Castel, 1995, p. 89). Au-delà du volet professionnel, cela s'applique également au problème du mariage.

6.Sources : Recensements 1976, 1986, 1996 (CAPMAS, Central Agency for Public Mobilisation And Statistics); Rapports sur le Développement humain 1981, 1998.

7.Entre 1960 et 1996, les taux de scolarisation primaire et secondaire combinés sont passés de $42 \%$ à $80 \%$ au niveau national et de $58,9 \%$ à $91,3 \%$ pour la ville du Caire. De même, entre 1986 et 1996, la durée moyenne de scolarité a augmenté de 3,7 années à 4,1 années en 1996 au niveau national, respectivement 5,8 années et 6,2 années au Caire. Enfin, et à titre d'exemple, 25,8\% des adultes âgés de plus de 15 ans étaient alphabétisés en 1960, 55,5 \% en 1996, pour l'ensemble du pays (respectivement 48,9 \% à $72,9 \%$ pour la ville du Caire).

8.Un quart des parents interviewés savent lire et écrire, quatre d'entre eux ont le niveau primaire (respectivement 3 pères et une mère) et deux pères ont achevé un cursus secondaire.

9.On pense ici à la généralisation des cours particuliers qui coûtent tous les ans 6 milliards Łe (soit 2 milliards \$US) aux familles égyptiennes, soit $4 \%$ du PNB en 1996 (Égypte Monde arabe, 2 et 3etrimestre 1996, n 27-28, p. 519).

10.Dans certains cas, l'arbitrage en faveur du secondaire professionnel et technique ou de l'apprentissage s'explique non seulement par des raisons financières, mais surtout parce qu'ils dispensent des compétences plus facilement monnayables sur le marché du travail. S'ajoute encore le fait que pour les parents, le marché du travail serait plus accessible aux jeunes munis d'un savoir-faire professionnel (Hoodfar, 1999). 11.La population active regroupe les individus en âge de travailler selon qu'ils sont actifs occupés ou chômeurs (individu n'ayant pas d'activité professionnelle, étant disponible sur le marché du travail et ayant entrepris des démarches). L'importance statistique des «nouveaux chômeurs » conduit le statisticien à les distinguer des " chômeurs ayant déjà travaillé » dans la saisie de la population active. La population inactive comprend les étudiants à temps plein, les personnes demeurant au foyer, celles ne cherchant pas d'emploi ou dans l'incapacité de travailler et les retraités ou nonretraités inactifs à cause de l'âge.

12.Le recensement de la population active et du chômage met en œuvre deux catégories intégrées à la population inactive : les personnes qui «ne cherchent pas d'emploi » (et qui par ailleurs ne sont pas étudiants ou « au foyer ») et celles qui sont «dans l'incapacité de travailler ». Dans le premier cas, sur 259902 personnes recensées, $59 \%$ sont âgées de 15 à 30 ans; dans le second, les 15-30 ans représentent $36 \%$ des 273 152 personnes se déclarant incapables de travailler. Les 20-30 ans représentent respectivement $30 \%$ et $22 \%$ de ces deux catégories. 
13.97 \% des jeunes âgés de 20 à 30 ans au chômage n'ont jamais travaillé (99 \% des jeunes femmes, 95\% des jeunes hommes).

$\mathbf{1 4 . 2 2} \%$ des jeunes âgés de 20 à 25 ans sont au chômage (38 \% des jeunes femmes et $17 \%$ des jeunes hommes). Pour les 25-30 ans, le taux de chômage est de $19 \%$

(respectivement $32 \%$ et $16 \%$ ).

15.Selon le recensement de 1996, l'emploi des femmes a doublé entre 1986 et 1996, passant ainsi de 1,39 million à 2,61 millions. En 1996, elles représentaient 15,2 \% de la population active (qui s'élevait à 17,1 millions) contre 10,9\% dix ans plutôt (des 12,8 millions de personnes âgées de 15 à 60 ans).

16.En 1996, les jeunes Égyptiens entendus comme les $15-30$ ans représentent $28 \%$ de la population. $66 \%$ de la population a moins de 30 ans. $49 \%$ en a moins de $20.25 \%$ des Égyptiens ont entre 10 et 19 ans.

17. Cette situation, avant tout conjoncturelle, devrait se résoudre par la diminution du nombre des demandeurs d'emploi. À plus long terme (2010-2020), l'inflexion de la croissance démographique devrait ainsi atténuer les besoins en termes de création d'emplois (Youssef, 2000, Courbage, 2000).

18. Ainsi, qu'il s'agisse de la migration vers les pays du Golfe de l'établissement d'une petite entreprise, les itinéraires professionnels des pères mobilisent à la fois leurs compétences professionnelles et les réseaux disponibles; et se comprennent comme des carrières professionnelles « objectives » (E. C. Hughes, 1996) centrées sur la réalisation d'un objectif professionnel ou matrimonial.

19.Dans ces trois cas, c'est précisément la « chance d'avoir un emploi stable, avec contrat, et qui soit décent » (Nermîne, 24 ans) qui oriente le projet professionnel. Cela ne signifie pas - notamment pour les deux hommes - que cet emploi soit leur seule activité. Elle demeure au centre de leur biographie professionnelle, mais leur journée de travail s'allonge au gré d'autres activités qui viennent fournir un supplément de revenus (qui n'est pas nécessairement orienté vers le financement du mariage). Stable sur le marché du travail formel, ils entrent et sortent ainsi régulièrement de l'économie informelle.

20.Il est nécessaire de préciser ici que nos premiers entretiens totalement construits sur la reconstitution de biographies professionnelles (où il n'était de notre part jamais question de mariage) ont immédiatement révélé l'importance du thème.

21.En termes de générations et pour l'ensemble de l'Égypte, l'âge au mariage serait passé de 18 ans à 20,2 ans entre les jeunes âgés de 25-29 ans et les 45-49 ans en 1995 (Courbage, 1999). Ainsi, en 30 ans (1962-1992), l'âge moyen au mariage serait passé de 20 à 22,4 ans (Courbage, 1995). Selon le Rapport sur le Développement humain (1998), l'âge moyen au premier mariage des femmes est passé de 19,8 ans en 1969 à 26,7 ans en 1996 (il est de 27,4 ans au Caire). Si ces dernières données paraissent refléter la situation urbaine, elles semblent excessives dans le contexte rural.

22.Dot payée par la famille du futur époux, elle est utilisée par la famille de la jeune femme pour financer la partie du mobilier qui lui incombe d'acheter.

23.Il faut « sauver les jeunes de la noyade » (al-Akhbâr, 15/07/99), alors que ces derniers " marchent sur le balcon » (cAmr, 25 ans, al-Gumhûriyya, 18/03/99) et sombrent dans la corruption et le marché noir (al-Nûr, 14/04/98; Rûz al-Youssef, 15/04/98) le satanisme (al-Wafd, 05/04/99), les drogues (al-Wafd, 12/09/99), à titre d'exemples...

24.Tourné Karine, 2000, "Le chômage, la création d'emploi et le temps libre ", Les écrits de Jeunesse, $\mathrm{n}^{\circ} 1$ (non publié, lettre d'information du programme " Jeunesses d'Égypte » - CEDEJ, disponible sur le site www.cedej.org.eg). 
25.Prévus pour encadrer les loisirs culturels et sportifs des jeunes, être des lieux d'échanges et d'information, les centres de jeunesses sont depuis quelques années au centre d'une polémique mettant en avant, d'une part, les abus et les dysfonctionnements (délabrement des locaux, corruption, délinquance...) et, d'autre part, l'effort du gouvernement pour améliorer la gestion de ces centres, leurs équipements et les services offerts aux jeunes.

26.Phénomène perçu d'abord comme « typiquement étudiant » (al-Hayât, 29/09/98; alWafd, 07/12/98 ; al-Gumhûriyya, 10/12/98, al-Ahrâr, 02/05/99) le mariage urfî (traduit le plus généralement par coutumier) est un mariage où le contrat est signé par les époux et deux témoins. «Il est plus souple et moins contraignant » (Sâmya, 25 ans), notamment d'un point de vue financier. Unis sans l'accord des parents, c'est pour les jeunes un moyen d'avoir des rapports sexuels. Pour les adultes, le mariage urfi est une forme "d'assurance pour les vieux jours ", notamment parmi les femmes veuves qui deviennent ainsi la deuxième épouse. (al-Wafd, 26/11/99).

27.Cité dans "Jeunesse en crise ", Revue de la presse égyptienne, CEROAC (Centre d'étude et de recherche sur l'Orient arabe contemporain), n 2, décembre 1981, p. 143.

\section{INDEX}

Mots-clés : jeunesse, sociologie, chômage

\section{AUTEUR}

KARINE TOURNÉ

Cedej 